\title{
Modeling the synergistic effects of toxicant mixtures
}

\author{
Matthias Liess ${ }^{1,2^{*}}$ (D), Sebastian Henz ${ }^{1,2}$ and Naeem Shahid ${ }^{1,2,3}$
}

\begin{abstract}
Background: Toxicants often occur simultaneously. Some combinations show synergistic combined effects that go far beyond what is predicted with current effect models. Up until now, only the combined additive effects of similar acting chemicals have been assessed accurately, whereas the combined effects of dissimilar acting chemicals have been greatly underestimated in many cases.

Results: Here, we use the individual tri-phasic concentration-response relationship of two toxicants with different modes of action to model their combined synergistic effect on Daphnia magna. The novel stress addition approach (SA) predicted the combined effects $\left(L C_{50}\right)$ of different esfenvalerate and prochloraz combinations with an uncertainty factor of 2.8 at most, while the traditional effect addition (EA) and concentration addition (CA) approaches underestimated the combined effect by a factor of up to 150 and 660, respectively. Data of the single substance concentration-response relationships and on their combined effects enable to determine the degree of synergism. For the evaluation of the combined toxicant effect, we provide the approach as R package and as Indicate model (http:// www.systemecology.eu/indicate/).

Conclusion: Adding stressors arithmetically, considering non-monotonic cause-effect relationships, is a decisive component in predicting the combined effects of multiple stressors within test systems. However, the extent of the synergistic effects that multiple stressors exert on populations within the ecosystem context is still highly controversial. Various processes are relevant at the ecosystem level, which are not considered in laboratory studies. However, the present work serves as a building block for understanding the effects of multiple stressors in the field.
\end{abstract}

Keywords: Multiple toxicants, Synergism, System stress, Stress addition model (SAM), Hormesis, Concentration addition, Effect addition

\section{Background}

In polluted ecosystems, a large number of toxicants usually occur simultaneously generating additive, antagonistic and synergistic effects. Some combinations show combined synergistic effects that go far beyond what is expected when using current effect models. This high degree of uncertainty makes it difficult to predict the biological effects of toxicant mixtures [1, 2]. For many

\footnotetext{
*Correspondence: matthias.liess@ufz.de

1 Dept. System-Ecotoxicology, UFZ-Helmholtz Centre for Environmental

Research, Permoserstrasse 15, 04318 Leipzig, Germany

Full list of author information is available at the end of the article
}

decades two concepts have been applied to predict the combined effects of toxicants:

(i) Combined effects of similar acting toxicants are usually assessed with the concentration addition approach (CA) [3]. The CA approach assumes that the concentrations of toxicants can be added if scaled by their toxicity. This combined effect prediction of toxicant mixtures with the same mode of action mostly provides satisfactory results, whereby even mixtures with 16 different substances can be accurately predicted [4].

(ii) Combined effects of dissimilar acting toxicants are generally assessed using the effect addition approach (EA) [5]. The EA approach assumes additivity of each of the two toxicant effects in terms of the probabilistic sum

\section{Springer Open}

(c) The Author(s) 2020. This article is licensed under a Creative Commons Attribution 4.0 International License, which permits use, sharing, adaptation, distribution and reproduction in any medium or format, as long as you give appropriate credit to the original author(s) and the source, provide a link to the Creative Commons licence, and indicate if changes were made. The images or other third party material in this article are included in the article's Creative Commons licence, unless indicated otherwise in a credit line to the material. If material is not included in the article's Creative Commons licence and your intended use is not permitted by statutory regulation or exceeds the permitted use, you will need to obtain permission directly from the copyright holder. To view a copy of this licence, visit http://creativeco mmons.org/licenses/by/4.0/. 
accounting for the probability that an individual is killed independently by each chemical and by subtracting the product of their effects.

However, predicting the combined effect of toxicant mixtures with different modes of action provides contradictory results. Examples of mostly successful predictions have been reviewed [6] and include the combined effects of up to 16 biocides on algae growth [7] and the effects on natural algal communities [8]. By contrast, a large number of investigations show that the combined effects of toxicants may reveal considerably greater effects than those predicted with the EA or the CA approach. Such unexpected effects are generally referred to as synergistic effects. Synergism is defined as substantially deviating from a selected null model, generally the CA or the EA approach [9]. A review of the interactions between pesticides in vertebrate studies reported that in some cases pesticide mixtures, particularly those involving insecticides, have been shown to be synergistic, with reported increases in toxicity of up to 100-fold [10]. Other groups of organisms also showed combined synergistic effects in both field and laboratory studies. These included bees [11-13], the bacteria Vibrio fischeri [14], and crustaceans $[14,15]$. Toxicant mixtures showing synergistic effects include cholinesterase inhibitors, pyrethroids, azole fungicides and antifoulants [16].

Similar to many toxicant mixtures, also environmental stressors in combination with pollutants may show synergistic effects [17-19]. However, also the combination of these types of stressors give contradictory results. For example, a meta-analysis showed that interactions were most frequently antagonistic: $40 \%$, compared with $26 \%$ synergistic; $19 \%$ additive and $15 \%$ reversed [20]. A further meta-analysis identified that only one of the two stressors had a significant effect in $39 \%$ of the analyzed cases, $28 \%$ of the paired-stressor combinations resulted in additive effects and 33\% resulted in interactive (antagonistic, synergistic, opposing or reversal) effects [21]. One approach to predict synergistic effects is the recently developed stress addition model (SAM) [22]. The SAM assumes that each individual organism has a general stress capacity towards all types of specific stressors. If this capacity is exceeded, the organism dies. Accordingly, the general stress is the common currency of any type of stress. The general stress levels of independent stressors are additive, with the sum determining the total stress exerted on a population. The approach provides a tool that quantitatively predicts the synergistic acute effects of independent stressor combinations. However, the SAM approach does not accurately predict the combined effects of stressors at low intensities, as the traditional logistic cause-and-effect relationship of individual stressors does not accurately reproduce the observed relationships.
The aim of this investigation is to provide an approach that models the synergistic effects of toxicant mixtures with different modes of action based on their individual effects also at low concentrations. The approach is based on the stress addition theory that has been successfully applied to predict the synergistic effects in organisms exposed to environmental stressors in combination with toxicants [22]. To identify single and combined effects of very low stress levels we consider non-monotone, tri-phasic concentration-response relationships with increasing survival at low toxicant levels [18].

\section{Material and methods \\ General}

We re-investigated an existing data set designed to study the combined effects of the insecticide esfenvalerate and the fungicide prochloraz under both low-food and highfood conditions [15]. Esfenvalerate, a pyrethroid, affects the nerve conduction by preventing the closure of the voltage-gated sodium channels in the axonal membranes [23]. In contrast, the azole fungicide prochloraz inhibits fungal growth by inhibiting cytochrome P450s [24]. In crustaceans, azole fungicides can also inhibit a wider range of cytochrome P450s [25], which participate in the detoxification of xenobiotic compounds including pyrethroids [26, 27]. The pesticides were selected for their different modes of action and their potential for synergistic combined effects to be modeled using the SA approach [22]. However, the modeling of the combined effects carried out so far showed large uncertainties in the low concentration range [15].

We set up a fully crossed factorial design with eight esfenvalerate treatments $(0,0.0001,0.001,0.01,0.1,0.316$, $1,3.16 \mu \mathrm{g} / \mathrm{L}) \times$ four prochloraz concentrations $(0,1,32$, $100 \mu \mathrm{g} / \mathrm{L}$ ) $\times$ two food levels (high, low). Details of the methods applied can be found in an earlier study [15]. In brief, during the test, the organisms used in the high food treatment were fed $0.5 \times 109$ cells per ind./day the first week, $1.15 \times 109$ cells per ind./day the second week, and $1.35 \times 109$ cells per ind./day the third and fourth weeks. In contrast, organisms in the low food treatment were fed with $0.5 \times 107$ cells per ind./day the first week, $1.15 \times 107$ cells per ind./day the second week, $1.35 \times 107$ cells per ind./day in the third and fourth weeks. The food dosage for low-food conditions was established according to preliminary range-finding tests that showed a minor effect on the survival of individuals (around 15\% as compared to high-food conditions) until the end of experiment (i.e., 4 weeks). Fecundity rates at the low-food condition were decreased (number of eggs per female over 21 days $=0.18$ ) as compared to high-food conditions (120), but were comparable to temporary food restrictions in the field [28] causing a decline in population 
density [29]. Before pesticide exposure, organisms were acclimatized to the corresponding food conditions for 7 days, were then exposed to the pesticides for $24 \mathrm{~h}$, and survival was monitored for an additional 21 days. For each treatment, we tested 15 daphnids with one individual per vessel containing $80 \mathrm{~mL}$ of the test solution in order to avoid toxicant-mediated competitive interactions between individuals [30]. The mortality of the daphnids was recorded daily and dead individuals were removed from the experiment. Neonates from each vessel were removed daily.

\section{Contaminants}

Both pesticides are frequently applied in agriculture as mixtures [31]. We used a combination of toxicants that is known to act synergistically compared to the null model of CA [14]. Fungicides that inhibit the synthesis of ergosterols have been proposed to be general synergists. The ergosterol-biosynthesis-inhibitors have been shown to enhance the effect of insecticides on various animals in both field and laboratory studies [10-12]. The tested concentrations of esfenvalerate, except for the highest concentrations ( 1 and $3.16 \mu \mathrm{g} / \mathrm{L}$ esfenvalerate), are in the range frequently detected in streams [32]. The tested low prochloraz concentrations are environmentally relevant, whereas $100 \mu \mathrm{g} / \mathrm{L}$ in particular does not occur in agricultural streams [32, 33]. We used dimethyl sulfoxide (DMSO) as a solvent for the preparation of the stock solutions and always kept concentrations below 0.02\% $[\mathrm{v} / \mathrm{v}]$ that is 2 orders of magnitude lower than the lowest observed-effect concentration (2\%) [34] and below the solvent limit suggested by the Organisation of Economic Cooperation and Development (OECD) guidelines [35].

\section{Chemical analysis of the test media}

Exposure concentrations of esfenvalerate and prochloraz were analyzed by Wessling GmbH, Landsberg OT, Oppin, Germany, using a Thermo Fisher Scientific TSQ 8000 Evo Triple Quadrupole GC - MS/MS. The median measured concentration of each nominal concentration ranged in acceptable boundaries $( \pm 20 \%)$. Details are provided in Shahid et al. 2019 [15]. Results in subsequent sections are displayed and analyzed using nominal concentrations.

\section{Test organisms}

In all experiments, we used D. magna individuals obtained from a clone "Aachen V" cultured at the Department System-Ecotoxicology, UFZ - Helmholtz Centre for Environmental Research, Leipzig, Germany.

\section{System stress (SyS) for single toxicant effects}

The concentration-response relationships of single substances were fitted with the $\mathrm{EC}_{\mathrm{x}-\mathrm{SyS}}$ model [18]. This approach fits a tri-phasic concentration-response relationship and also reveals effects at ultra-low toxicant concentrations.

The three assumptions are: (i) Individuals develop internal System stress (SyS) that may cause the mortality of some individuals within a population; (ii) external stress in the form of toxicant exposure reduces SyS. Accordingly, two opposing monotone relationships are assumed: toxicant stress increases with increasing toxicant concentration and SyS decreases with increasing pulsed toxicant stress (Fig. 1b); (iii) all independent stressors present-including SyS and toxicant stress are additive according to the "Stress Addition Model" (SAM) with the sum of the general stress determining the total stress exerted on an individual within a population [22]. In short, with the SAM, we assume that each individual has a general stress capacity towards all types of specific stress that should not be exhausted. Experimental stress levels are transferred into general stress levels of the SAM using the stress-related mortality as a common link. These general stress levels of independent stressors are additive, with the sum determining the total stress exerted on a population. A detailed description of the SAM can be found in Liess et al. 2016 [22]. We assume that SyS is an expression of the potential to adapt to a changing environment. This would suggest that individuals are evolutionarily "optimized" for a certain amount of short-term stress reflecting the "natural" stress level. The amount of stress at which SyS is reduced close to zero may therefore be the level of stress typically present in the environment of an individual [18].

\section{Multi-TOX approach}

In comparison to the null-model of concentration addition (CA) [3] or effect addition (EA) [5] the stress addition approach (SA, calculated with the SAM [22]) predicts the synergistic effects of independent stressors. The combined effect of toxicants is calculated as follows:

i. For each single substance the toxicant stress and system stress relations are calculated using the $\mathrm{EC}_{\mathrm{x} \text {-SyS }}$ model [18]. This approach considers a triphasic concentration-response relationship that differs from the log-logistic relationship, especially in the range of low concentrations.

ii. Toxicants may act partly similarly and also partly independently. In order to calculate the combined effect of toxicant combinations that show a mixture of both modes of action, the toxicant stress was determined using a combination of SA and CA. The toxicant stress according to SA is calculated by the sum of the toxicant stresses of both pesticides: 

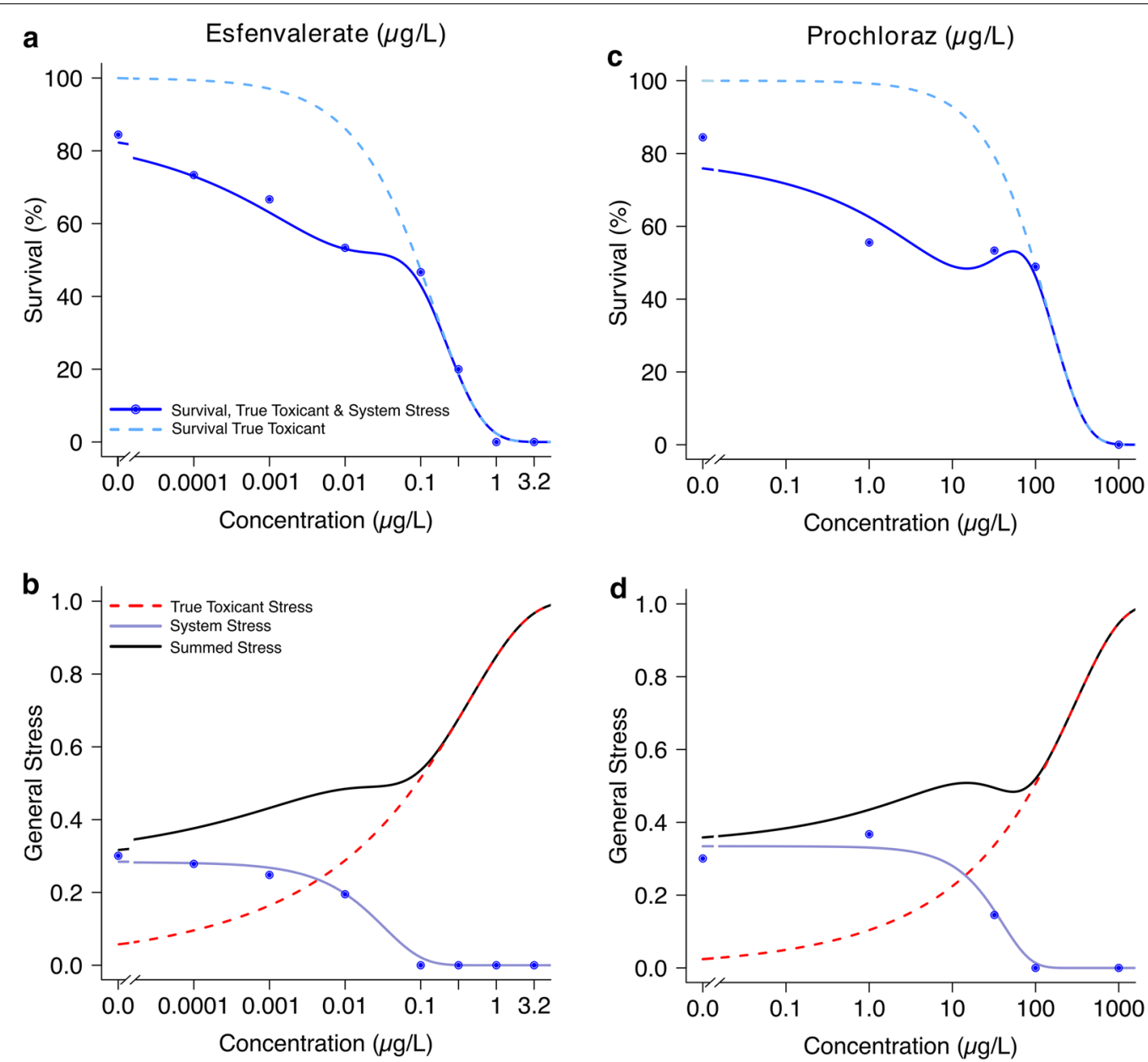

Fig. 1 Single substance concentration-response. Observed and modeled survival-response and stress-response relationships of Daphnia magna at low-food conditions for day 21 after $24 \mathrm{~h}$ of esfenvalerate and prochloraz exposure. a, c Observed mortality (dots), modeled mortality applying the $\mathrm{EC}_{\mathrm{x} \text {-Sys }}$ model (line), toxicant-related mortality (dashed line). b, d Modeled toxicant-stress, system-stress, summed stress

$$
S_{\text {tox }, \mathrm{SA}}=S_{\text {tox }, \mathrm{E}}+S_{\text {tox }, \mathrm{P}}
$$

where $S_{\text {tox }, \mathrm{E}}$ and $\mathrm{S}_{\text {tox, } \mathrm{P}}$ are the toxicant stresses of esfenvalerate and prochloraz [18].

For the calculation of the toxicant stress according to $\mathrm{CA}$, the concentration of prochloraz is first converted into an equivalent esfenvalerate concentration via the true toxicant-survival curve that does not include SyS (Fig. 1). This is then added to the concentration of esfenvalerate. Using the modeled true toxicant-survival curve of esfenvalerate, the survival corresponding to this concentration sum is determined and converted into a general stress value $\left(\mathrm{S}_{\text {tox,CA,E}}\right)$. This process is asymmetric because the curve shapes of esfenvalerate and prochloraz are different. To compensate for this, the steps are also carried out in the other direction, converting the esfenvalerate concentration into an equivalent prochloraz concentration, resulting in a different general stress $\left(\mathrm{S}_{\text {tox,CA,P}}\right)$. Both results are then averaged:

$$
S_{\text {tox, } \mathrm{CA}}=\frac{S_{\text {tox }, \mathrm{CA}, \mathrm{E}}+S_{\text {tox }, \mathrm{CA}, \mathrm{P}}}{2}
$$

iii. The total toxicant stress of the mixture is calculated by interpolating the SA and CA toxicant stress, whereby SA and CA may contribute in different proportions to the result:

$$
S_{\mathrm{tox}}=S_{\mathrm{tox}, \mathrm{CA}} \cdot P_{\mathrm{CA}}+S_{\mathrm{tox}, \mathrm{SA}} \cdot P_{\mathrm{SA}},
$$

where $\mathrm{P}_{\mathrm{CA}}$ and $\mathrm{P}_{\mathrm{SA}}$ are the proportions of concentration addition and stress addition. We propose that a toxicant mixture can act partially similar and partially dissimilar. The SA toxicant stress represents the dissimilar acting part, whereas the $\mathrm{CA}$ 
toxicant stress shows the similar acting part. We identified these proportions by minimizing the root mean squared error (RMSE) between the observed and predicted effects. Only after the identification of these proportions for each toxicant combination, a prediction of combined effects is possible.

iv. According to the $\mathrm{EC}_{\mathrm{x} \text {-SyS }}$ model, an increase in the toxicant stress decreases the system stress. We use this negative correlation to calculate the reduced System Stress as a result from the combined toxicant stress, $\mathrm{S}_{\text {tox,CA}}$. This combined toxicant stress was calculated according to the concentration addition approach as the empirical evidence indicates that both toxicants act together according the CA concept to reduce the system stress. The calculation is carried out for both pesticides because the correlation between SyS and toxicant stress is different for each pesticide. After that, both SyS are averaged:

$$
S_{\text {SyS }}=\frac{S_{\text {SyS,E }}+S_{\text {SyS,P }}}{2}
$$

with $S_{\text {SyS }}$ being the total system stress of the mixture and $S_{S y S, E}$ and $S_{S y S, P}$ the system stresses of esfenvalerate and prochloraz.

v. Finally, the total toxicant stress $\left(\mathrm{S}_{\text {tox }}\right)$ and the total SyS $\left(\mathrm{S}_{\text {SyS }}\right)$ are combined, resulting in the total general stress $(\mathrm{S})$ :

$$
S=S_{\text {tox }}+S_{\text {SyS }}
$$

This total stress is then converted using a beta distribution into the predicted effect of the pesticide mixture. Any additional long-term environmental stress that may exist is not separately included in the calculation of stressors. In the present investigation, this is food stress that was administered already before the organisms were exposed to toxicant stress. Such long-term stress may trigger adaptation processes in the organism. Adaptation and recovery are not implicitly considered within the current approach. Accordingly, the current Multi-Tox approach may not be suitable for experiments including very high environmental stress.

\section{$\mathrm{LC}_{50}$ of the observed effects}

For the calculation of the $\mathrm{LC}_{50}$, we fitted the measured data with the $\mathrm{EC}_{\mathrm{x} \text {-SyS }}$ model as described in Liess et al. 2019 [18] using the $\mathrm{EC}_{\mathrm{x} \text {-SyS }}$ module version 2019.11 in Indicate 1.1.1. This approach enables realistic $\mathrm{LC}_{50}$ values to be estimated even in the presence of a tri-phasic concentration-response relationship.

\section{Traditional methods for predicting the effects of pesticide} combinations

For the concentration addition approach [3] the prochloraz concentration is converted to an equivalent esfenvalerate concentration using mortality as a link. In other words, a prochloraz concentration which causes a certain mortality is equivalent to an esfenvalerate concentration which causes the same mortality. This allows the consideration of substance-specific concentrationresponse relationships for the toxicants in the mixture. The converted prochloraz concentration-the esfenvalerate-equivalent prochloraz concentration-is then added to the esfenvalerate concentration and converted into an effect using the concentration-response relationship of esfenvalerate. For the effect addition (EA) approach the effects are added according to the equation by Bliss (1939) [5]:

$$
E_{\mathrm{mix}}=1-\prod_{i=1}^{n}\left(1-E_{i}\right)
$$

where $E_{\text {mix }}$ is the total effect and $E_{i}$ are the effects of the individual toxicants. The CA and EA methods described here are the same as in Liess 2016 [22]. The calculations were carried out using the SAM module version 2019.10 in Indicate version 1.1.1.

Comparisons of whole cause-effect relationships were quantified by the root mean square error (RMSE) which is the square root of the average of the squared differences between observed and predicted values [36]. Comparisons of point estimates $\left(\mathrm{LC}_{50}\right)$ were quantified by the model deviation ratio (MDR), which was calculated by dividing the predicted toxicity by the observed toxicity [6].

\section{Results}

\section{Single toxicant effects}

The concentration-survival curves for the two individual toxicants in Fig. 1 show the esfenvalerate-related survival (Fig. 1a), toxicant and system stress (Fig. 1b) as well as prochloraz-related survival (Fig. 1c), toxicant and system stress (Fig. 1d); (high food, Additional file 1: Fig. S1). The concentration-response relationships for both toxicants, displayed in Fig. $1 \mathrm{~A}$ and $\mathrm{C}$ deviated from the simplified log-logistic relationship especially at medium concentrations. A "step" at these concentrations characterizes the tri-phasic relationship as described by Liess et al. 2019 [18]. Accordingly, the concentration-response relationships were fitted with the $\mathrm{EC}_{\mathrm{x} \text {-SyS }}$ model [18] that allows to separately identify the toxicant and system stress (Fig. 1b, d). The strength of these stressors is used in the next step to calculate the combined toxicant effects. 


\section{Combined toxicant effects}

The combination of esfenvalerate and prochloraz acts highly synergistically. This experimentally observed combined effect on Daphnia magna was modeled on the basis of the concentration-response relationship of each pesticide using 3 different approaches: (i) the MultiTOX approach presented here with the basic assumption of stress addition (SA); (ii) the traditional concentration addition (CA) and (iii) the effect addition (EA). A comparison of the experimental results with the predictions of the three models shows that for both food levels the Multi-TOX approach generally predicts the measurement results considerably better than the CA and EA approaches (low food: Fig. 2; high food Additional file 1: Fig S2). This applies to the course of the entire curve determined by the root mean square error (RMSE) as well as to the $\mathrm{LC}_{50}$ (Table 1 ). However, at very low prochloraz concentrations of $1 \mu \mathrm{g} / \mathrm{L}$ (2-3 orders of magnitude below its $\mathrm{LC}_{50}$ ), no major synergistic interactions of both toxicants were observed. In this range of low concentrations all of the models investigated allow a good prediction of the combined toxicity (Fig. 2a).

\section{Food restriction}

The shape of the concentration-response relationships for the two substances alone, for the combined effects of both toxicants and for both, high and low food levels can in all cases be described by a combined stress function: with increasing concentration an increasing toxicant stress and a decreasing system stress result in a tri-phasic concentration-effect relationship. A differentiated assessment of the results shows that food restriction leads to an increased sensitivity of individuals. In addition, food restriction with simultaneous exposure to 2 toxins causes an increased synergistic effect in the test organisms (Fig. 2, Additional file 1: Fig. S2; Table 1). This synergistic effect is only slightly underestimated by the SA approach with a factor of 2.8; the traditional EA and CA approaches, however, underestimate the combined effect by a factor of 150 and 660, respectively (Table 1 ).

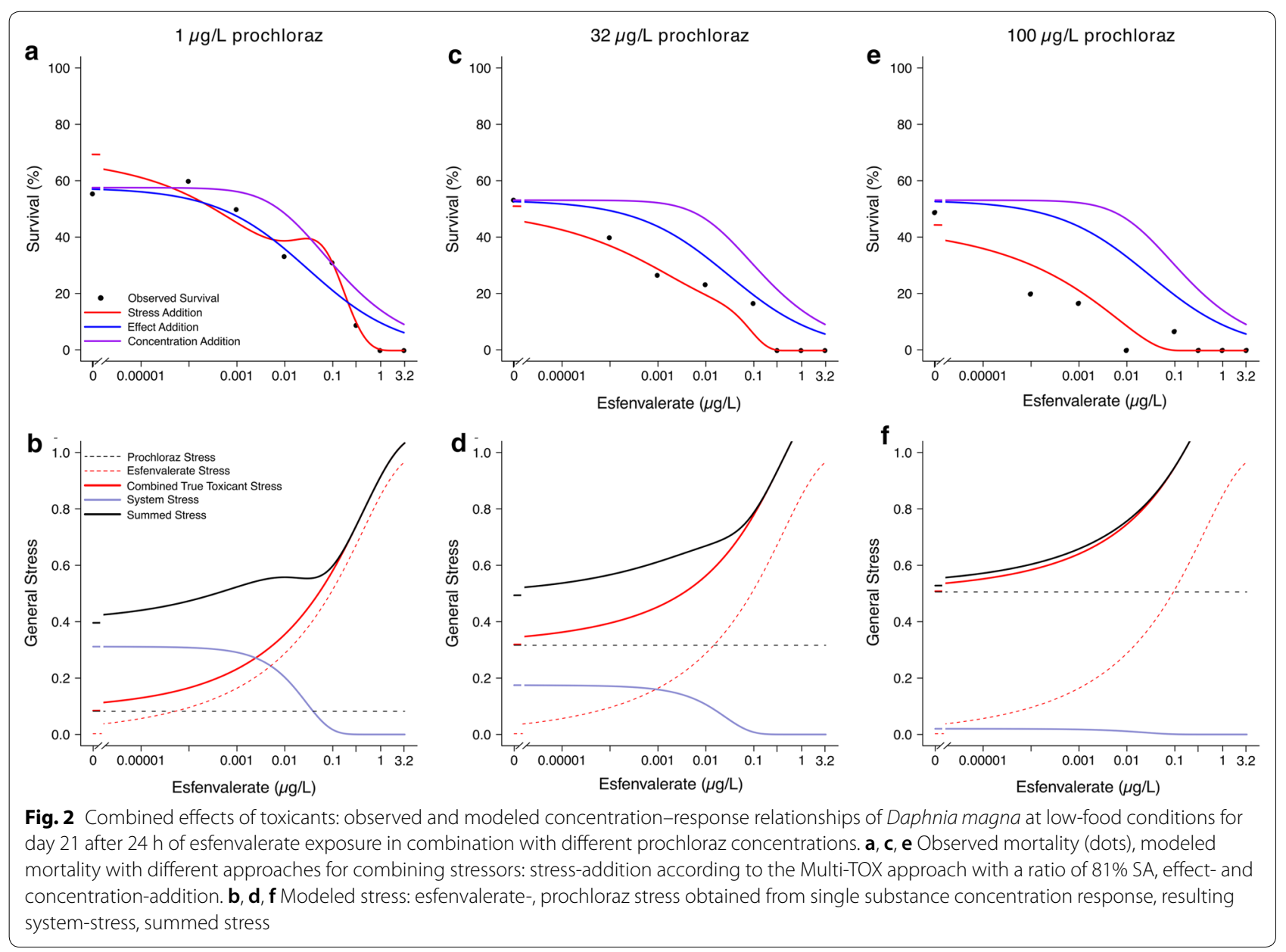


Table 1 Quantification of the fit between the observed and predicted combined effect of the toxicant: experimental observations and predictions of toxicant effect on Daphnia magna exposed to esfenvalerate alone and in combination with prochloraz concentrations under high- and low-food conditions

\begin{tabular}{|c|c|c|c|c|c|c|c|c|c|c|}
\hline \multirow[t]{2}{*}{ Time point } & \multirow[t]{2}{*}{ Food level } & \multirow{2}{*}{$\begin{array}{l}\text { Prochloraz } \\
(\mu \mathrm{g} / \mathrm{L})\end{array}$} & \multicolumn{3}{|c|}{ RMSE } & \multirow{2}{*}{$\begin{array}{l}\text { Observed }^{b} \\
\mathrm{LC}_{50}\end{array}$} & \multirow{2}{*}{$\begin{array}{l}\text { Predicted }^{\mathrm{c}} \\
\mathrm{LC}_{50}\end{array}$} & \multicolumn{3}{|c|}{ MDR $^{d}$} \\
\hline & & & $S A^{a}$ & $C A$ & EA & & & $S A^{a}$ & $C A$ & $\mathrm{EA}$ \\
\hline \multirow[t]{8}{*}{ Day 21} & Low food & 0 & - & - & - & 0.098 & - & - & - & - \\
\hline & & 1 & 6.0 & 8.3 & 5.6 & 0.048 & 0.079 & 1.7 & 3.2 & 0.93 \\
\hline & & 32 & 4.2 & 17 & 10 & 0.0066 & 0.0023 & 0.34 & 25 & 6.8 \\
\hline & & 100 & 5.8 & 25 & 18 & 0.0003 & 0.0008 & 2.8 & 660 & 150 \\
\hline & High food & 0 & - & - & - & 0.49 & - & - & - & - \\
\hline & & 1 & 6.8 & 3.1 & 3.1 & 0.55 & 0.59 & 0.77 & 0.94 & 0.94 \\
\hline & & 32 & 8.4 & 17 & 16 & 0.19 & 0.21 & 1.4 & 2.8 & 2.6 \\
\hline & & 100 & 4.9 & 14 & 13 & 0.20 & 0.22 & 1.0 & 2.7 & 2.6 \\
\hline \multirow[t]{8}{*}{ Day 7} & Low food & 0 & - & - & - & 0.13 & - & - & - & - \\
\hline & & 1 & 5.9 & 9.7 & 6.8 & 0.053 & 0.11 & 2.1 & 2.8 & 1.4 \\
\hline & & 32 & 4.1 & 15 & 9.8 & 0.012 & 0.017 & 1.4 & 14 & 6.2 \\
\hline & & 100 & 7.7 & 25 & 19 & 0.001 & 0.0032 & 3.2 & 240 & 75 \\
\hline & High food & 0 & - & - & - & 0.53 & - & - & - & - \\
\hline & & 1 & 5.6 & 4.4 & 4.4 & 0.65 & 0.49 & 0.80 & 0.82 & 0.82 \\
\hline & & 32 & 8.0 & 14 & 14 & 0.26 & 0.33 & 1.2 & 2.1 & 2.0 \\
\hline & & 100 & 7.4 & 13 & 13 & 0.25 & 0.19 & 0.71 & 2.2 & 2.1 \\
\hline
\end{tabular}

Predictions with different approaches for combining stressors: Stress-Addition according to the Multi-TOX approach (SA), effect-addition (EA) and concentrationaddition (CA). Whole curve estimates of combined toxicant effect: root mean square error (RMSE). Point estimate ( $L C_{50}$ ) of combined toxicant effect: model deviation ratio (MDR)

a SA (stress addition) calculated according to the Multi-TOX approach. For low food $81 \%$ SA, for high food 38\% SA

b Observed $\mathrm{LC}_{50}$ calculated with the mean survival of all experimental repetitions, fitted with $\mathrm{EC}_{\mathrm{x} \text {-SyS }}$

c Predicted $\mathrm{LC}_{50}$ based on the concentration-response relationships of single toxicants modeled with stress addition according to the Multi-TOX approach

$d$ MDR values are calculated by dividing the predicted $L_{50}$ of $S A, C A$, EA by the observed $L C_{50}$. For values of CA $>2$, interactions between stressors are interpreted as synergistic related to $C A$

\section{Discussion}

\section{The Multi-TOX approach}

Our aim was to provide an approach that models the synergistic effects of toxicant mixtures with different modes of action based on their individual effects also at low concentrations. The key challenge in such modeling is to identify a convertible "common currency" that quantifies the strength of the stress exerted by different toxins. Only this enables to move from the identification of case-specific relationships to the development of generally applicable mechanistic models. The concept of general stress is used for this purpose as it provides a "common currency" for calculating the combined effect of two toxins [22]. Furthermore, the exact quantification of the stress exerted by each individual toxicant is of great importance for predicting the combined effects of several stressors. Especially in low concentration ranges, even the low stress of individual toxicants can potentiate the combined effect of several stressors [22]. Just how important the exact quantification of concentration-effect relationships is became clear in a mixing experiment where the individual substances did not show monotonous but hormesis-like concentration-effect relationships [8]. Here, the concept of "System Stress" (SyS) provides a substantial improvement by enabling the fit of nonmonotone dose-response models [18]. For example, in the present publication with the consideration of SyS the MDR after 7 days for all low-food experiments is on average 2.2; in contrast to a significantly worse MDR of 105 without consideration of SyS as in Shahid et al. (2019) [15]. Even after 21 days, the MDR in the current publication was only 2.5 , whereas without taking SyS into account, a meaningful calculation of the MDR was not possible [15].

The Multi-Tox approach presented here can also be applied when the specific mechanisms of combined effects are unknown. That is different to the substanceand species-specific approach used so far that allows a deeper understanding of the respective mechanisms. Examples include (i) the combined effect of clothianidin and propiconazole where propiconazole caused a prolonged emergence time indicative of a reduced detoxification capacity [13]; (ii) the synergistic potential of prochloraz on several pesticides that was proposed to be 
due to an effect on P450 monooxygenase enzymes active in pesticide metabolism in these organisms [14]. However, more generic approaches were suggested to predict the biological effects of the virtually unlimited number of possible stressor combinations. In this area, an approach was proposed which focused on bioenergetic alterations to assess the effects and ecological consequences of combined environmental stressors [37]. The basis for the concept of energy-limited tolerance to stress [38] integrating physiological [37] and population-level endpoints as provided by the dynamic energy budget (DEB) model [39]. With such a bio-energetic approach, the higher toxicity of many pesticides under increasing mean temperatures and daily temperature fluctuations could be associated with a reduced cellular energy allocation [40]. Nevertheless, the question remains as to how these sublethal responses can be integrated to predict individual mortality in natural conditions, the most integral-and hence pivotal-endpoint when assessing the fitness of individuals and higher levels of biological organization.

\section{Factors determining the synergy strength}

Here we show that a mixture of two dissimilar acting chemicals acts synergistically in relation to CA. Also other studies identified synergistic combined effects of dissimilar acting chemicals: examples include investigations on the combined effect of prochloraz, azoxystrobin, diquat and esfenvalerate on Daphnia magna and the bacterium Vibrio fischeri [14] and the combined effect of alpha-cypermethrin, and prochloraz, propiconazole and epoxiconazole on D. magna [41]. However, also various studies satisfactorily describe the combined effect of dissimilar acting chemicals with the EA or the CA approach without observing synergistic combined effects. Examples include investigations on the combined effects of binary mixtures of similar- and dissimilar-acting toxicants on Daphnia magna [42], 16 biocides with different mechanisms of action that were administered to algae during one generation cycle (24 h) [7] and a mixture of six dissimilarly acting substances and their effects on carbon fixation in natural algal communities [8]. Obviously, dissimilar acting chemicals do not cause synergistic combined effects in all cases. Accordingly, the question arises as to which factors increase the occurrence of synergistic combined effects. Firstly, acute tests with a very short duration exhibit a lower synergistic effect of mixtures of dissimilar toxicants than chronic tests. Examples include (i) increased synergy between a pyrethroid insecticide and azole fungicides in a 14-day test with Daphnia magna compared to an 48-h acute toxicity test [41] and (ii) synergy between a pyrethroid insecticide and the fungicides propiconazole and prochloraz in a 4-day test with Daphnia magna compared to an 7-h acute toxicity test
[31]. Secondly, food shortage increased the synergistic effect between stressors. Examples include the increased synergism on Daphnia magna tested with a mixture of a pyrethroid insecticide and azole fungicide [15], but also the synergistic effects between toxicants and environmental stressors: the combined effects of copper and UV radiation on the crustacean Paramorea walkeri [17] is considerably stronger at low-food levels. In the present study, both factors favoring synergism were present: long observation time compared to the life span of D. magna and food restriction. The authors expect that under such conditions also other mixtures of dissimilar substances exhibit increased synergistic effects, independently of the substance-specific mechanism of synergism.

\section{Relevance for risk assessment}

The presence of a large number of different toxicants in the field, coupled with frequently occurring environmental stress, could potentially result in a large number of synergistic toxicant combinations-contributing to the "Effect Paradox": unexpectedly high sensitivity of some field populations [43]. Accordingly, determining the synergistic potential of frequently occurring toxicant mixtures will provide insight about the processes determining the sensitivity of field populations. This requires experimental data of the single substance concentration-response relationship with sufficient concentration tested, especially in the range of low effects. Additionally, information on the combined effects of mixturescarried out under realistic environmental conditions, including food limitation, are needed to determine the ratio/degree of synergism. The resulting information will facilitate the identification of chemical traits that in combination act synergistically. For the evaluation of the combined toxicant effect, we provide the Multi-TOX approach as $\mathrm{R}$ package "stressaddition" available from CRAN and as an Indicate model (download: https:// www.systemecology.eu/indicate/).

\section{Conclusion}

Here we provide an approach that mechanistically quantifies the synergistic effects of toxicant mixtures with different modes of action. To identify single and combined effects at very low stress levels, we also consider non-monotone, tri-phasic concentrationresponse relationships [18]. This possibility of adding stressors arithmetically, considering non-monotone cause-effect relationships, is a decisive component in predicting the combined effects of multiple stressors within test systems. Nevertheless, the question remains to the extent of the synergistic effects that multiple stressors exert on populations within the ecosystem 
context. Empirical evidence is still highly controversial. Ranging from investigations that found little evidence for the importance of interactions among stressors to shape macroinvertebrate communities [44] towards studies that identified strong interactive effects between pesticides and environmental stressors, e.g., high water temperature [45] or reduced stream connectivity [46]. Various processes are relevant at the ecosystem level, which are not considered in laboratory studies [43]. However, the present work is a building block for understanding the effects of multiple stressors in the field.

\section{Supplementary information}

Supplementary information accompanies this paper at https://doi. org/10.1186/s12302-020-00394-7.

Additional file 1. Additional figures.

\section{Abbreviations}

SA: Stress addition; EA: Effect addition; CA: Concentration addition; SAM: Stress addition model; SyS: System stress; EC $_{x-\text { Sys: }}$ Effect concentration modeled under consideration of system stress; RMSE: Root mean square error; MDR: Model deviation ratio; DEB: Dynamic energy budget; CRAN: Comprehensive R Archive Network.

\section{Acknowledgements}

The authors thank the System-Ecotoxicology team for their critical discussions on this topic. We would also like to thank 4 reviewers for their valuable input to improve the manuscript.

\section{Authors' contributions}

ML conceived the approach and wrote the paper. SH programmed and optimized the SA-model and programmed the R-package. NS performed the laboratory experiments and contributed to the optimization of the SA-model. All authors read and approved the final manuscript.

\section{Funding}

This work was supported by the Helmholtz long-range strategic research funding (POF III) and the German Academic Exchange Service (Deutscher Akademischer Austauschdienst, DAAD) for financially supporting N.S. through doctoral fellowship.

\section{Availability of data and materials}

Data and combined toxicity models are available as the $R$ package "stressaddition" available from CRAN and as an Indicate file (App download: http://www. systemecology.eu/indicate/).

\section{Ethics approval and consent to participate}

Not applicable.

\section{Consent for publication}

Not applicable.

\section{Competing interests}

The authors declare that they have no competing interests.

\section{Author details}

${ }^{1}$ Dept. System-Ecotoxicology, UFZ-Helmholtz Centre for Environmental Research, Permoserstrasse 15,04318 Leipzig, Germany. ${ }^{2}$ Institute for Environmental Research (Biology V), RWTH Aachen University, Worringerweg 1 , 52074 Aachen, Germany. ${ }^{3}$ Department of Environmental Sciences, COMSATS University Islamabad, Vehari Campus, Vehari 61100, Pakistan.
Received: 5 June 2020 Accepted: 27 August 2020

Published online: 19 September 2020

\section{References}

1. Frische T, Egerer S, Matezki S et al (2018) 5-Point programme for sustainable plant protection. Environ Sci Eur. https://doi.org/10.1186/s1230 2-018-0136-2

2. Schäfer RB, Liess M, Altenburger R et al (2019) Future pesticide risk assessment: narrowing the gap between intention and reality. Environ Sci Eur 31:21. https://doi.org/10.1186/s12302-019-0203-3

3. Loewe S, Muischnek H (1926) Über Kombinationswirkungen 1. Mitteilung: Hilfsmittel der Fragestellung. In: Naunyn-Schmiedebergs Arch Exp Pathol Pharmakol. pp 313-326

4. Altenburger R, Backhaus T, Boedeker W et al (2000) Predictability of the toxicity of multiple chemical mixtures to Vibrio fischeri: mixtures composed of similarly acting chemicals. Environ Toxicol Chem. https://doi. org/10.1002/etc.5620190926

5. Bliss Cl (1939) The toxicity of poisons applied jointly. Ann Appl Biol 26:585-615

6. Belden JB, Gilliom RJ, Lydy MJ (2007) How well can we predict the toxicity of pesticide mixtures to aquatic life? Integr Environ Assess Manag 3:364372. https://doi.org/10.1897/1551-3793(2007)3[364:HWCWPT]2.0.CO;2

7. Faust $M$, Altenburger R, Backhaus T et al (2003) Joint algal toxicity of 16 dissimilarly acting chemicals is predictable by the concept of independent action. Aquat Toxicol. https://doi.org/10.1016/S0166-445X(02)00133 $-9$

8. Backhaus T, Arrhenius A, Blanck H (2004) Toxicity of a mixture of dissimilarly acting substances to natural algal communities: Predictive power and limitations of independent action and concentration addition. Environ Sci Technol 38:6363-6370. https://doi.org/10.1021/es0497678|ISSN0 013-936X

9. Schäfer RB, Piggott JJ (2018) Advancing understanding and prediction in multiple stressor research through a mechanistic basis for null models. Glob Chang Biol 24:1817-1826. https://doi.org/10.1111/gcb.14073

10. Thompson HM (1996) Interactions between pesticides; a review of reported effects and their implications for wildlife risk assessment. Ecotoxicology 5:59-81. https://doi.org/10.1007/BF00119047

11. Meled M, Thrasyvoulou A, Belzunces LP (1998) Seasonal variation in susceptibility of Apis mellifera to the synergistic action of prochloraz and deltamethrin. Environ Toxicol Chem. https://doi.org/10.1897/15515028(1998)017<2517:svisoa>2.3.co;2

12. Schmuck R, Stadler T, Schmidt HW (2003) Field relevance of a synergistic effect observed in the laboratory between an EBI fungicide and a chloronicotinyl insecticide in the honeybee (Apis mellifera L, Hymenoptera. Pest Manag Sci. https://doi.org/10.1002/ps.626

13. Teper D, Sgolastra F, Bosch J et al (2018) Combined exposure to sublethal concentrations of an insecticide and a fungicide affect feeding, ovary development and longevity in a solitary bee. Proc R Soc B Biol Sci 285:20180887. https://doi.org/10.1098/rspb.2018.0887

14. Cedergreen N, Kamper A, Streibig JC (2006) Is prochloraz a potent synergist across aquatic species? A study on bacteria, daphnia, algae and higher plants. Aquat Toxicol 78:243-252. https://doi.org/10.1016/j.aquat ox.2006.03.007

15. Shahid N, Liess M, Knillmann S (2019) Environmental stress increases synergistic effects of pesticide mixtures on Daphnia magna. Environ Sci Technol 53:12586-12593. https://doi.org/10.1021/acs.est.9b04293

16. Cedergreen N (2014) Quantifying synergy: a systematic review of mixture toxicity studies within environmental toxicology. PLoS ONE 9:96580. https ://doi.org/10.1371/journal.pone.0096580

17. Liess M, Champeau O, Riddle M et al (2001) Combined effects of ultraviolet-B radiation and food shortage on the sensitivity of the Antarctic amphipod Paramoera walkeri to copper. Environ Toxicol Chem 20:2088-2092

18. Liess M, Henz S, Knillmann S (2019) Predicting low-concentration effects of pesticides. Sci Rep 9:1-9. https://doi.org/10.1038/s41598-019-51645-4

19. Relyea RA, Mills N (2001) Predator-induced stress makes the pesticide carbaryl more deadly to gray treefrog tadpoles (Hyla versicolor). Proc Natl Acad Sci USA 98:2491-2496. https://doi.org/10.1073/Pnas.031076198 
20. Jackson MC, Loewen CJG, Vinebrooke RD, Chimimba CT (2016) Net effects of multiple stressors in freshwater ecosystems: a meta-analysis. Glob Chang Biol 22:180-189. https://doi.org/10.1111/gcb.13028

21. Birk S, Chapman D, Carvalho L et al (2020) Impacts of multiple stressors on freshwater biota across spatial scales and ecosystems. Nat Ecol Evol. https://doi.org/10.1038/s41559-020-1216-4

22. Liess M, Foit K, Knillmann S et al (2016) Predicting the synergy of multiple stress effects. Sci Rep 6:32965. https://doi.org/10.1038/srep32965

23. Silver KS, Du Y, Nomura Y, et al (2014) Voltage-gated sodium channels as insecticide targets. In: Advances in Insect Physiology

24. Copping LG, Hewitt HG (1998) Chemistry and mode of action of crop protection agents. R Soc Chem. https://doi.org/10.1039/9781847550422

25. Gottardi M, Kretschmann A, Cedergreen N (2016) Measuring cytochrome P450 activity in aquatic invertebrates: a critical evaluation of in vitro and in vivo methods. Ecotoxicology. https://doi.org/10.1007/s1064 6-015-1600-z

26. Bach J, Snegaroff J (1989) Effects of the fungicide prochloraz on xenobiotic metabolism in rainbow trout: In vivo induction. Xenobiotica. https:// doi.org/10.3109/00498258909034671

27. Pilling ED, Bromleychallenor KAC, Walker CH, Jepson PC (1995) Mechanism of synergism between the pyrethroid insecticide $\lambda$-cyhalothrin and the imidazole fungicide prochloraz, in the honeybee (Apis mellifera L.). Pestic Biochem Physiol. https://doi.org/10.1006/pest.1995.1001

28. Tessier AJ (1986) Comparative population regulation of two planktonic Cladocera (Holopedium gibberum and Daphnia catawba). Ecology. https ://doi.org/10.2307/1938573

29. Müller-Navarra D, Lampert W (1996) Seasonal patterns of food limitation in Daphnia galeata: separating food quantity and food quality effects. J Plankton Res 18:1137-1157. https://doi.org/10.1093/plankt/18.7.1137

30. Liess M (2002) Population response to toxicants is altered by intraspecific interaction. Environ Toxicol Chem 21:138-142. https://doi.org/10.1002/ etc.5620210120

31. Kretschmann A, Gottardi M, Dalhoff K, Cedergreen N (2015) The synergistic potential of the azole fungicides prochloraz and propiconazole toward a short alpha-cypermethrin pulse increases over time in Daphnia magna. Aquat Toxicol 162:94-101. https://doi.org/10.1016/j.aquatox.2015.02.011

32. Münze R, Hannemann C, Orlinskiy P et al (2017) Pesticides from wastewater treatment plant effluents affect invertebrate communities. Sci Total Environ 599-600:387-399

33. Kreuger J (1998) Pesticides in stream water within an agricultural catchment in southern Sweden, 1990-1996. Sci Total Environ 216:227-251

34. Bowman MC, Oiler WL, Cairns T et al (1981) Stressed bioassay systems for rapid screening of pesticide residues. Part l: evaluation of bioassay systems. Arch Environ Contam Toxicol. https://doi.org/10.1007/BF01057571
35. OECD (2002) Guidance document on aquatic toxicity testing of difficult substances and mixtures. OECD Publishing, Paris

36. Aptula AO, Jeliazkova NG, Schultz TW, Cronin MTD (2005) The better predictive model: High q2 for the training set or low root mean square error of prediction for the test set? QSAR Comb Sci. https://doi.org/10.1002/ qsar.200430909

37. Pörtner $\mathrm{HO}$ (2012) Integrating climate-related stressor effects on marine organisms: Unifying principles linking molecule to ecosystem-level changes. Mar Ecol Prog Ser. https://doi.org/10.3354/meps10123

38. Sokolova IM (2013) Energy-limited tolerance to stress as a conceptual framework to integrate the effects of multiple stressors. Integr Comp Biol. https://doi.org/10.1093/icb/ict028

39. Kooijman SALM (2000) Dynamic Energy and Mass Budgets in Biological Systems

40. Verheyen J, Stoks R (2020) Negative bioenergetic responses to pesticides in damselfly larvae are more likely when it is hotter and when temperatures fluctuate. Chemosphere. https://doi.org/10.1016/j.chemospher e.2019.125369

41. Bjergager MBA, Dalhoff K, Kretschmann A et al (2017) Determining lower threshold concentrations for synergistic effects. Aquat Toxicol 182:79-90. https://doi.org/10.1016/j.aquatox.2016.10.020

42. Syberg K, Elleby A, Pedersen $\mathrm{H}$ et al (2008) Mixture toxicity of three toxicants with similar and dissimilar modes of action to Daphnia magna. Ecotoxicol Environ Saf. https://doi.org/10.1016/j.ecoenv.2007.05.010

43. Liess M, Ratte T, Ebke P, Hollert H (2019) 20 years SETAC GLB: increasing realism of pesticide risk assessment. Environ Sci Eur 31:13. https://doi. org/10.1186/s12302-019-0197-x

44. Kath J, Thomson JR, Thompson RM et al (2018) Interactions among stressors may be weak: Implications for management of freshwater macroinvertebrate communities. Divers Distrib 24:939-950. https://doi. org/10.1111/ddi.12737

45. Russo R, Becker JM, Liess M (2018) Sequential exposure to low levels of pesticides and temperature stress increase toxicological sensitivity of crustaceans. Sci Total Environ 610:563-569. https://doi.org/10.1016/j.scito tenv.2017.08.073

46. Knillmann S, Orlinskiy P, Kaske $O$ et al (2018) Indication of pesticide effects and recolonization in streams. Sci Total Environ 630:1619-1627. https:// doi.org/10.1016/j.scitotenv.2018.02.056

\section{Publisher's Note}

Springer Nature remains neutral with regard to jurisdictional claims in published maps and institutional affiliations.

\section{Submit your manuscript to a SpringerOpen ${ }^{\circ}$ journal and benefit from:}

- Convenient online submission

- Rigorous peer review

- Open access: articles freely available online

- High visibility within the field

- Retaining the copyright to your article

Submit your next manuscript at springeropen.com 